\title{
Fabrication of Hybrid Diamond and Transparent Conducting Metal Oxide Electrode for Spectroelectrochemistry
}

\author{
Jingping Hu, James Hodge, Arthur J. Boff, and John S. Foord \\ Chemistry Research Laboratory, Department of Chemistry, University of Oxford, Mansfield Road, Oxford OX1 3TA, UK \\ Correspondence should be addressed to John S. Foord, john.foord@chem.ox.ac.uk
}

Received 10 February 2011; Accepted 24 February 2011

Academic Editor: Bengi Uslu

Copyright () 2011 Jingping Hu et al. This is an open access article distributed under the Creative Commons Attribution License, which permits unrestricted use, distribution, and reproduction in any medium, provided the original work is properly cited.

\begin{abstract}
A novel diamond transparent electrode is constructed by integrating conductive diamond film and transparent conducting metal oxide to combine the superior electrochemical properties of diamond and the electrical conductivity of transparent metal oxide (TCO). Direct growth of diamond on indium tin oxide (ITO) and aluminium doped zinc oxide (AZO) was explored, but X-ray photoelectron spectroscopy measurement reveals that both substrates cannot survive from the aggressive environment of diamond growth even if the latter is regarded as one of the most stable TCO. As a second route, a diamond membrane in silicon frame was prepared by selective chemical etching, and a diamond optically transparent electrode (OTE) was constructed by assembling the diamond membrane on the top of an ITO-coated substrate. The resulting device exhibits a high optical transparency and quasireversible electrochemical kinetics, which are competitive to other diamond OTEs reported previously. Its application in UV-Vis spectroelectrochemical studies on the oxidisation of 4-aminophenol was demonstrated.
\end{abstract}

\section{Introduction}

The development of optically transparent electrodes (OTEs) for electrochemical and particularly spectroelectrochemical studies has received considerable attention for investigation of electrochemical reaction mechanism and electroanalytical applications [1]. By measuring the transmission or reflection of UV, visible, or IR light through the electrode, the spectra of electrogenerated species can be characterised directly. In additional to the cell design, the choice of OTE is a focus of investigation. Conventionally, the OTEs are consisted of metal $(\mathrm{Au}, \mathrm{Pt})[2,3]$ or metal oxide thin films deposited on transparent substrates (such as glass or quartz). The use of metal micromech and minigrid was also reported $[4,5]$.

Boron doped diamond (BDD) shows a combination of unique properties, such as a wide potential window, a low background current, mechanical and chemical stability, and resistance to fouling [6]. The application of BDD as OTEs in spectroelectrochemical studies was reported previously. Zak and Haymond et al. demonstrated the concept using a mechanical polished free-standing diamond of $0.38 \mathrm{~mm}$ thickness for the detection of ferricyanide, methyl viologen [7], and ferrocene [8]. BDD thin films of $0.5 \mu \mathrm{m}$ and $1 \mu \mathrm{m}$ thickness were deposited on quartz from microwave-assisted chemical vapour deposition (MWCVD) and used to characterise the electrochemical responsiveness of $\mathrm{Ru}\left(\mathrm{NH}_{3}\right)_{6}^{3+}$, $\mathrm{Fe}(\mathrm{CN})_{6}^{3-}$, and chlorpromazine [9]. A 100-mesh platinum grid was coated with BDD thin film as an OTE, and applied to the chronoabsorptometry measurement of the redox couples, $\mathrm{Rb}(\mathrm{bpy}){ }_{3}^{3+}$ and $\mathrm{Fe}(\mathrm{phen})_{3}^{3+}[10]$. The application of BDD OTE requires two seemingly contradictory features, optical transparency and electrical conductivity. Higher boron doping is desired to increase electrical conductivity, but the boron inclusion creates electronic and vibrational states within the bandgap, leading to strong light absorption. So, a compromise must be reached to balance the doping levels required for electrical conductivity $\left(R_{s}\right)$ while maintaining sufficient optical transparency $(T)$, which is described by the figure of merit $\Phi_{\mathrm{TC}}=T / R_{s}[11]$.

To achieve a good transparency as well as an improved electrical conductivity, we report a novel approach to fabricate diamond OTEs by integrating diamond thin film with a more conductive transparent substrate, such as indium tin oxide (ITO) and aluminium doped zinc oxide (AZO). The underlying conductive substrate will carry the majority of the currents, and the device resistance is mediated by 


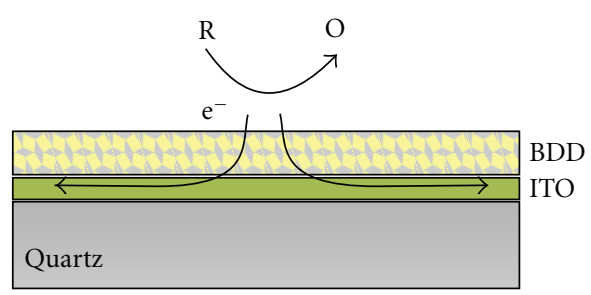

(a)

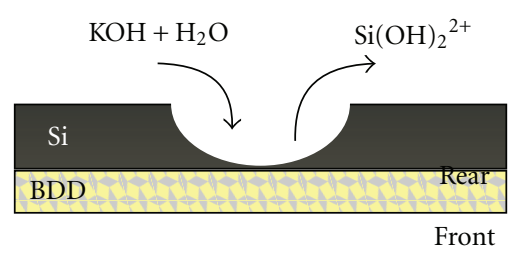

(b)

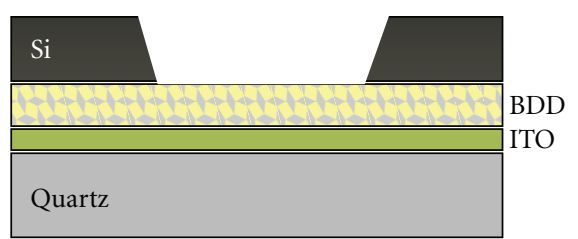

(c)

FIGURE 1: (a) Redox reaction on BDD surface and electron transfer through BDD membrane and along ITO film. (b) Chemical etching of silicon support to prepare free-standing BDD membrane. (c) Hybrid structure of diamond membrane-ITO electrode.

the resistance through the thin diamond film and the sheet resistance of the underlying highly conductive ITO substrate, which is much more conductive than the large sheet resistance along the diamond film in the conventional structure. This hybrid structure also permits the BDD thin film to be only lightly doped and hence highly transparent. In this work, two approaches were explored to fabricate this hybrid OTE, direct CVD deposition of BDD film on top of ITO or AZO thin films, or integration of a framesupported diamond membrane with an ITO electrode. The feasibility of the former method was explored by investigating the chemical stability of ITO and AZO film in harsh diamond growth conditions. The device from the latter route was characterised by spectroscopic, optical, and electrochemical measurements, and its application in UV-Vis spectroelectrochemical studies on 4 -aminophenol was also demonstrated.

\section{Experimental}

2.1. Fabrication of Diamond/ITO or AZO OTEs. An ITO film of $400 \mathrm{~nm}$ thickness was deposited on fused silica quartz substrate by electron beam evaporation of premixed powder of $\mathrm{In}_{2} \mathrm{O}_{3}$ with $10 \% \mathrm{SnO}_{2}$ in $1.5 \times 10^{-4}$ mbar of oxygen. The deposition was followed by annealing in ambient conditions at $400^{\circ} \mathrm{C}$ for 2 hours. The metallic indium particles will be oxidised to $\mathrm{In}_{2} \mathrm{O}_{3}$ by atmospheric oxygen, and the associated defects are eliminated by annealing [12]. After annealing, the colour changed from dark grey to light green tint, and the transmission is over $90 \%$ between $450 \mathrm{~nm}$ and $900 \mathrm{~nm}$ in UV-Vis spectra. AZO film was prepared by the sol gel process [13]. A mixture of zinc acetate $\left(\mathrm{Zn}\left(\mathrm{CH}_{3} \mathrm{CO}_{2}\right)_{2} \cdot 2 \mathrm{H}_{2} \mathrm{O}\right)$ and aluminium nitrate $\left(\mathrm{Al}\left(\mathrm{NO}_{3}\right)_{3} \cdot 9 \mathrm{H}_{2} \mathrm{O}, 0.8\right.$ at.\%) was refluxed in 2-propanol with equimolar diethanolamine (DEA) and two molar equivalents of water at $78^{\circ} \mathrm{C}$ for 3 hours to get a clear solution, where DEA and added water acted as solstabiliser [14]. After aging for three days, the sol was used for spin coating on fused silica quartz substrate. The AZOcoated samples were annealed at $530^{\circ} \mathrm{C}$ in air for 30 minutes to improve the crystallinity and performance [13].

The ITO- or AZO-coated quartz substrate was ultrasonically seeded in nanodiamond $(3.5 \mathrm{~nm}$, Yorkshire bioscience Ltd, UK) slurries for half an hour before diamond deposition. The diamond film was deposited by hot filament assisted chemical vapour deposition (HFCVD) from a mixture of hydrogen $(99.3 \%)$ and methane $(0.7 \%)$ gases. Boron doping was achieved by passing hydrogen gas through an ice-cooled bubbler containing trimethylborate $(99 \%$, Sigma-Aldrich Ltd). The HFCVD was conducted using a tungsten filament above $2200^{\circ} \mathrm{C}$ on a substrate heated to around $800^{\circ} \mathrm{C}$ in a vacuum chamber at around 33 mbar. The structure of the OTE with an electron transfer route is illustrated in Figure 1(a).

2.2. Fabrication of Diamond Membrane/ITO Composite OTEs. $\mathrm{N}$-type (100) silicon substrate of $655 \mu \mathrm{m}$ thickness was ultrasonically seeded in a mixture of $10 \mu \mathrm{m}$ and $250 \mathrm{~nm}$ diamond suspension for one hour. A mixture of slurry was employed to enhance the nucleation due to "hammering effect" [15]. After seeding, the silicon substrate was rinsed with copious amounts of water to remove the excess diamond debris and dried with compressed air. Boron doped diamond was deposited from an HFCVD reactor under similar conditions for ITO and AZO substrates, except that the growth time is around 10 hours to achieve a mechanical robust diamond membrane. The diamond film coated silicon was sealed in a purposely built PTFE cell, leaving only a circular area in the rear side of Si substrate exposed. The exposed silicon was selectively chemical etched in $30 \% \mathrm{KOH}$ held in a water bath of $80^{\circ} \mathrm{C}$ to prepare diamond membrane, as illustrated in Figure 1(b). The etching rate is around $67 \mu \mathrm{m}$ per hour, and the etching time is around 10 hours. The membrane thickness is estimated to be $11.8 \mu \mathrm{m}$ from the weight of delaminated BDD membranes. 


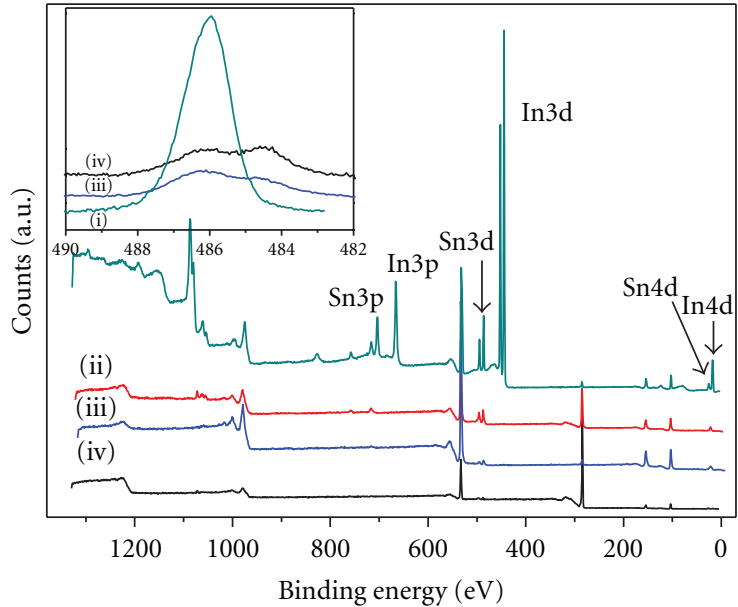

(a)

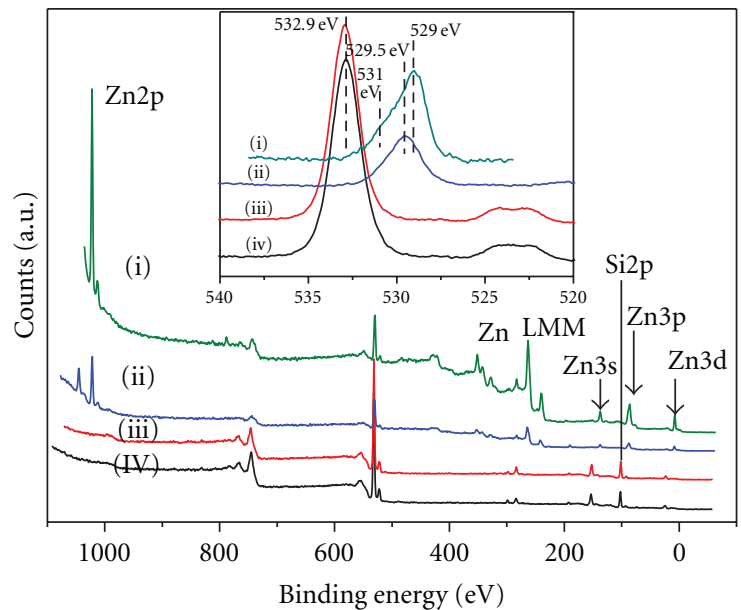

(b)

FIGURE 2: (a) XPS survey scan and $S n 3 \mathrm{~d}_{5 / 2}$ spectra (inset) of (i) as-grown ITO film, (ii) after 30 minutes diamond growth on ITO substrate, (iii) after 1 hour hydrogen plasma treatment at $800^{\circ} \mathrm{C}$, (iv) after two hours diamond growth on ITO substrate. (b) XPS survey scan and O1s spectra (inset) of (i) as-prepared AZO film, (ii) after 5 minutes and (iii) 10 minutes hydrogen plasma treatment at $800^{\circ} \mathrm{C}$ in an $\mathrm{HFCVD}$ reactor, (iv) after 30 minutes diamond growth on AZO substrate.

The diamond membrane in silicon frame was assembled onto a $400 \mathrm{~nm}$ thick ITO film on fused silica quartz substrate, as shown in Figure 1(c). The device was further insulated with nail varnish and epoxy, and an electrical connection was made by attaching an aluminium foil on the edge of ITO film. The diamond membrane and ITO formed an electrical contact due to surface tension after a wetting and drying process.

2.3. Characterisation of Diamond OTEs. The surface chemical state was characterised by an in-house built X-ray photoelectron spectroscopy (XPS) with an $\mathrm{Mg} \mathrm{K}_{\alpha} \mathrm{X}$-ray source and a high resolution XPS system (Scienta ESCA 300) with an $\mathrm{Al} \mathrm{K}_{\alpha} \mathrm{X}$-ray source in National Centre for Electron Spectroscopy and Surface Analysis (NCESS) at Daresbury Laboratory, UK. The film morphology was measured by NanoScope MultiMode AFM (Digital Instrument) in contact mode, and Raman spectra were recorded using a Dilor Raman spectroscopy with an Ar laser source (514.5 nm). The optical transparency was characterised by a fibre optic UVVis spectrometer (USB2000, Ocean Optics Inc.) equipped with deuterium and halogen lamps and a Unicam UV2 scanning spectrophotometer.

Electrochemical experiments were performed using an Autolab PSTAT 10 bipotentiostat (EcoChemie, Netherlands) in a PTFE one-compartment electrochemical cell, incorporating the diamond OTE as a working electrode, a platinum counter electrode, and a standard calomel reference electrode. Spectroelectrochemical measurements were performed using a special designed thin-layer cell. Hexaamineruthenium $\left(\mathrm{Ru}\left(\mathrm{NH}_{3}\right)_{6}^{3+}, 98 \%\right)$ and 4-aminophenol $(98 \%)$ were ordered from Aldrich and used as redox couples without further purification. Acetate buffer of $0.1 \mathrm{M}$ was used as supporting electrolyte for all electrochemical measurements and prepared using Milli-Q water $(>18 \mathrm{M} \Omega \mathrm{cm})$. All solutions were freshly prepared and deaerated by bubbling with argon gas before each electrochemical experiment.

\section{Results and Discussion}

3.1. Direct CVD Growth of BDD on ITO or AZO Electrodes. BDD was grown directly on ITO-coated quartz substrate, and the deposition process was characterised by XPS, as shown in Figure 2(a). Before diamond deposition, the asgrown ITO film (Figure 2(a)(i)) shows intense and sharp peaks of In, Sn, and $\mathrm{O} 1 \mathrm{~s}$ ascribed to indium oxide and tin oxide comprising the ITO film, together with weak peaks of $\mathrm{Si} 2 \mathrm{p}$ and $\mathrm{Si} 2 \mathrm{~s}$ originated from the quartz substrate, confirming the phase purity of the film. After diamond growth or hydrogen plasma treatment, no indium peaks can be discernable from the background any more, and the $\mathrm{Sn}$ peaks diminishes drastically with longer treatment time, as seen from Figure 2(a)(ii)-(iv). Although the increase of $\mathrm{C} 1 \mathrm{~s}$ peak intensity with diamond growth time indicates the presence of diamond film on the surface, the absence and diminishing of In and Sn peaks are not attributed to the burying of ITO by diamond layer, as diamond layer is still incomplete which, is evidenced by the presence of $\mathrm{Si}$ $2 \mathrm{p}$ and $\mathrm{Si} 2 \mathrm{~s}$ peaks from quartz substrate as presented in Figure 2(a)(ii).

The inset of Figure 2(a)(i, iii, iv) shows the $\operatorname{Sn} 3 \mathrm{~d}_{5 / 2}$ peaks of as-grown ITO, ITO after one hour hydrogen plasma treatment, and after two hours diamond growth, respectively. After plasma treatment and diamond growth, the sharp Sn $3 \mathrm{~d}_{5 / 2}$ peak of $\mathrm{SnO}$ at $486.10 \mathrm{eV}$ diminishes, accompanied by the appearance and growth of another peak attributed to elemental tin at $484.4 \mathrm{eV}$ with longer treatment time. This reflects that both indium oxide and tin oxide were reduced to elemental metals in the HFCVD reactor by hydrogen plasma 
due to high concentration of hydrogen radicals at elevated temperature. Most of the reduced elemental indium and tin evaporated rapidly at elevated substrate temperature under diamond growth conditions, and the former evaporated faster than the latter due to a lower melting temperature $\left(156^{\circ} \mathrm{C}\right)$ and a higher vapour pressure. This explanation is consistent with the literature that metal agglomeration was observed by SEM after hydrogen plasma treatment, and the figure of merit decreases more than $80 \%$ after one minute treatment by hydrogen plasma at $200^{\circ} \mathrm{C}$ [16]. Similar phenomenon has been observed that the emergence of elemental Sn $3 d_{5 / 2}$ peak was observed in XPS measurement after exposing in hydrogen plasma of $0.25 \mathrm{~W} / \mathrm{cm}^{2} \mathrm{rf}$ power density at $250^{\circ} \mathrm{C}$ for 30 minutes [17]. It is also reported that annealing of reduced ITO films in air at $400^{\circ} \mathrm{C}$ for 1 hour can restore to a complete oxidised state, which is, however, not observed in our case as almost all the reduced indium and tin metals are evaporated due to the elevated temperature $\left(800^{\circ} \mathrm{C}\right)$ and intense plasma power $(100 \mathrm{~W})$ in an HFCVD reactor.

Since ITO cannot survive from the aggressive diamond growth environment, a more stable substrate is preferred for the fabrication of a hybrid OTE. The previous study shows that zinc oxide is much more resistant to hydrogen plasma reduction, photodecomposition, and is more thermal stable at higher temperature than other TCOs [13, 17-19]. So a direct diamond growth on zinc oxide film was also explored. Figure 2(b) shows the XPS spectra of as-prepared AZO films from sol-gel process, and the XPS spectra after hydrogen plasma treatment of 5 and 10 minutes and after 30 minutes diamond growth, respectively. The as-prepared AZO film is characterised by strong $\mathrm{Zn} 2 \mathrm{p}$ core lines and LMM Auger peaks in the survey spectrum (Figure 2(b)-i). The aluminium peak is so weak and not detectable due to a low doping level. It can be clearly seen that the zinc signal diminishes, and Si 2 p core line from quartz substrate appears after exposure to hydrogen plasma at $800^{\circ} \mathrm{C}$, indicating the decomposition of AZO film. The as-prepared AZO film shows a main peak at $529.0 \mathrm{eV}$, as shown in Figure 2(b), attributable to the $\mathrm{O}^{2+}$ ions of $\mathrm{O}-\mathrm{Zn}$ bonding surrounded by $\mathrm{Zn}$. The broad shoulder at higher binding energies can be attributed to the loosely bound oxygen species on the surface or hydrated oxides [20,21], probably originating from acetate in the sol-gel process. During the hydrogen plasma treatment, hydrogen adsorbs on the surface forming $\mathrm{Zn}-\mathrm{H}$ and $\mathrm{OH}$ bonds [22], and adsorbed hydrogen can also be bonded in bridged structures between two zinc ( $\mathrm{Zn}-\mathrm{H}$ $\mathrm{Zn})$ or oxygen atoms $(\mathrm{OH} \cdots \mathrm{O})$. The formation of such surface bonds leads to surface passivation which makes $\mathrm{ZnO}$ stable towards thermal decomposition and hydrogen plasma reduction. The passivation layer has a slightly higher binding energy, which explains the shift of O1s peak towards higher binding energy after 5 minutes in hydrogen plasma, as presented in Figure 2(b). With a prolonged exposure to hydrogen plasma, the O1s peak shifts to a much higher binding energy of $532.9 \mathrm{eV}$ which is attributed to $\mathrm{SiO}_{2}$ from quartz substrate. We observed an unstable $\mathrm{ZnO}$ film under the harsh conditions for diamond growth, in contrary to the good stability of $\mathrm{ZnO}$ film upon mild hydrogen plasma treatment observed by Major et al. [17], probably due to different experimental conditions such as temperature and plasmas power.

3.2. A Hybrid BDD OTE with a BDD Membrane on ITO. Since the first route of directly diamond deposition was undermined by the stability of ITO and AZO substrate, we explored an alternative method to circumvent the problem by preparing silicon frame-supported diamond membrane separately followed by integrating diamond membrane with an ITO conducting substrate to fabricate the OTE device.

The morphology of the as-prepared diamond membrane was examined by AFM in contact mode, as shown in Figures 3(a) and 3(b). Optical application of OTE requires a smooth surface with a grain size much smaller than the wavelength of incident light to eliminate light scattering. To achieve this, the silicon substrate was ultrasonic treated in mixed micro- and nanodiamond slurries to increase the nucleation density. Following the ultrasonic treatment, the deposited diamond film exhibits a surface root mean square (RMS) roughness of around $26 \mathrm{~nm}$ and a grain diameter between 50 to $100 \mathrm{~nm}$, which corresponds to a nucleation density of $1 \times 10^{10}$ to $4 \times 10^{10} \mathrm{~cm}^{-2}$. The features of surface morphology confirm the significance of the ultrasonic treatment process.

The diamond phase on both front and rear side of membrane was characterised by Raman spectroscopy, as presented in Figure 4(a). The characteristic peaks of $\mathrm{sp}^{3}$ carbon at $1333 \mathrm{~cm}^{-1}$ are weak and broad in both spectra, indicating dense grain boundaries inherit to small diamond grains. The peaks at $1130 \mathrm{~cm}^{-1}$ and around $1470 \mathrm{~cm}^{-1}$ are most likely associated with transpolyacetylene at grain boundaries which is often observed in nanodiamond. The peak at $1130 \mathrm{~cm}^{-1}$ is less pronounced on the rear side of membrane, probably due to the inclusion of $\mathrm{SiC}$ in the nucleation phase of diamond growth and the modification during chemical etching. The peaks centred at around $1345 \mathrm{~cm}^{-1}$ and $1550 \mathrm{~cm}^{-1}$ are attributed to $\mathrm{sp}^{2}$ amorphous carbon which seems intense due to a resonant enhancement of $\mathrm{sp}^{2}$ bonded portion by a factor of 50 to 200 [23].

The optical properties of the OTE fabricated from diamond membrane were characterised by UV-Vis spectroscopy measurement, as illustrated in Figure 4(b). The optical transmittance of the diamond OTE is around $27 \%$ between 600 and $850 \mathrm{~nm}$. The falloff of transmission near and in the UV region is due to light scattering associated with surface roughness. This transmittance value is much higher than the free-standing diamond OTE reported by Zak et al. [7] which has best transmittance of $5 \%$ at around $300 \mathrm{~nm}$ and $1 \%$ at $600 \mathrm{~nm}$, and comparable to the diamond coated quartz OTE and diamond membrane fabricated by Stotter et al. [9] and Michaelson et al. [24]. In comparison with the OTE, the ITO film is highly transparent and has marginal contribution to the absorption, with an average transmittance around $95 \%$ at the interference fringes. Besides absorption by diamond membrane, light reflection by the smooth diamond membrane and ITO film might also contribute to the transmission loss. 


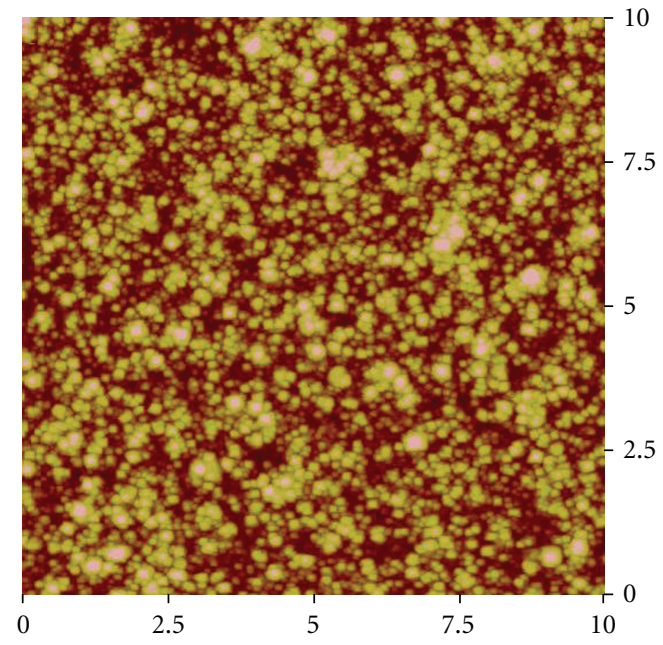

(a)

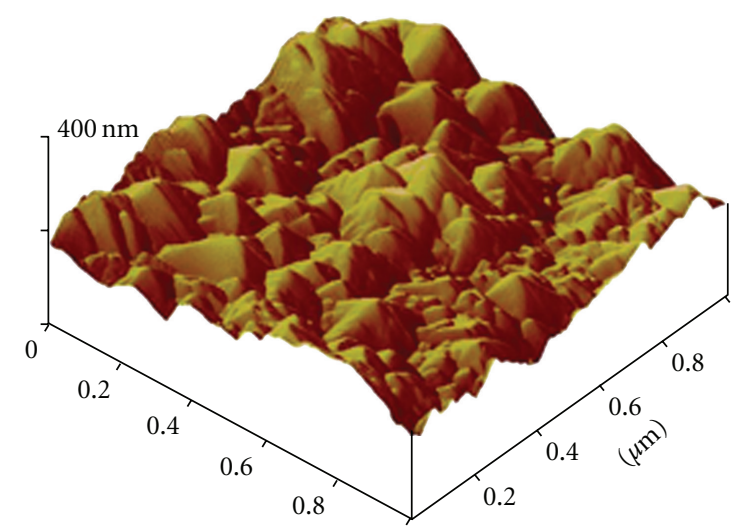

(b)

Figure 3: AFM images of diamond membrane, (a) $1 \times 1 \mu \mathrm{m}$ and (b) $10 \times 10 \mu \mathrm{m}$. The diameter of diamond grain is around 50 to $100 \mathrm{~nm}$, and the root mean square (RMS) roughness is around $26 \mathrm{~nm}$.

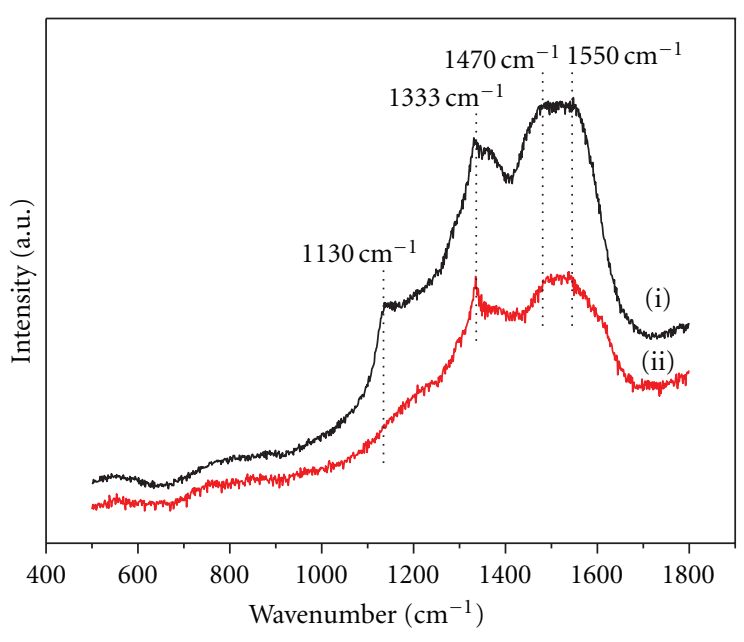

(a)

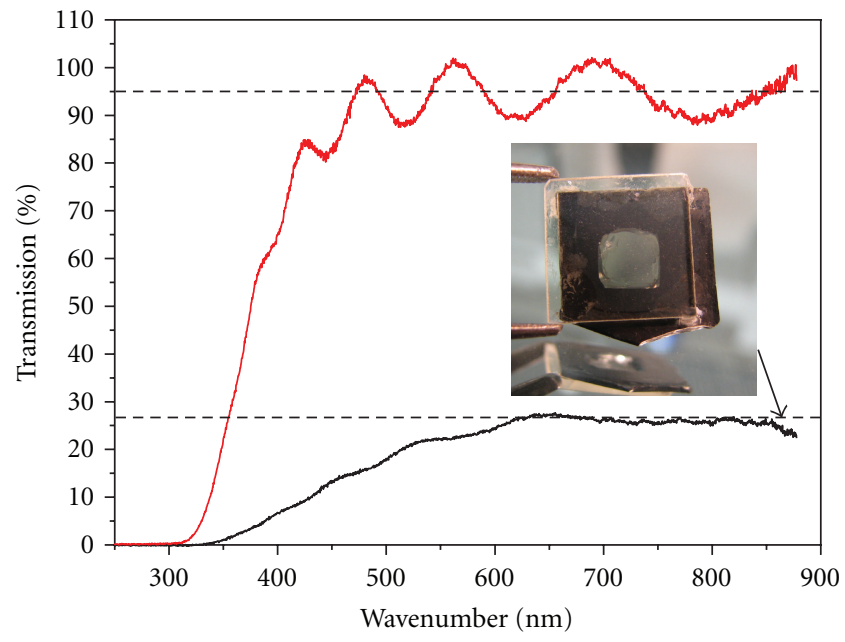

(b)

FIgURE 4: (a) The Raman spectra of (i) front side and (ii) rear side of diamond membrane. (b) The UV-Vis transmission spectra of ITO and fabricated OTE device consisting of a diamond membrane on an ITO substrate.

3.3. Electrochemical Properties of Hybrid OTE Device: BDD Membrane on ITO. The background corrected electrochemical response of the diamond OTE is measured by cyclic voltammetry in $\mathrm{Ru}\left(\mathrm{NH}_{3}\right)_{6}^{3+}$ of different concentrations in $0.1 \mathrm{M}$ acetate buffer as a supporting electrolyte as shown in Figure 5(a). The voltammograms have well-defined redox peaks, and a wide potential window from -1.35 to $1.50 \mathrm{~V}$ (Figure 5(b)), which is consistent with the value reported for free-standing diamond and diamond/quartz OTEs [7, 9]. A small peak separation is a characteristic of fast and reversible electron transfer kinetics. The diamond hybrid OTE exhibits a peak separation of 100 to $170 \mathrm{mV}$ depending on the analyte concentration (Figures 5(a)-5(c)), much smaller than the value reported for free-standing diamond OTE of $0.38 \mathrm{~mm}$ thickness in $1 \mathrm{mM} \mathrm{Fe}(\mathrm{CN})_{6}^{4-}$ [7], and comparable to the value for diamond/quartz OTEs in $0.1 \mathrm{mM}$ ferrocene and CPZ $[8,9]$. The peak separation of diamond hybrid OTE increases, as shown in Figure 5(c), and the curve tends to converge to $77 \mathrm{mV}$ at close to zero analyte concentration which is close to the typical value for Nernstian systems, demonstrating the quasireversible behaviour of our OTEs. The linear relationship between peak separation and analyte concentration also suggests that the uncompensated resistance is responsible for the relative large peak separation instead of sluggish electron transfer kinetics. Besides resistances from solution and electrical contacts, the 


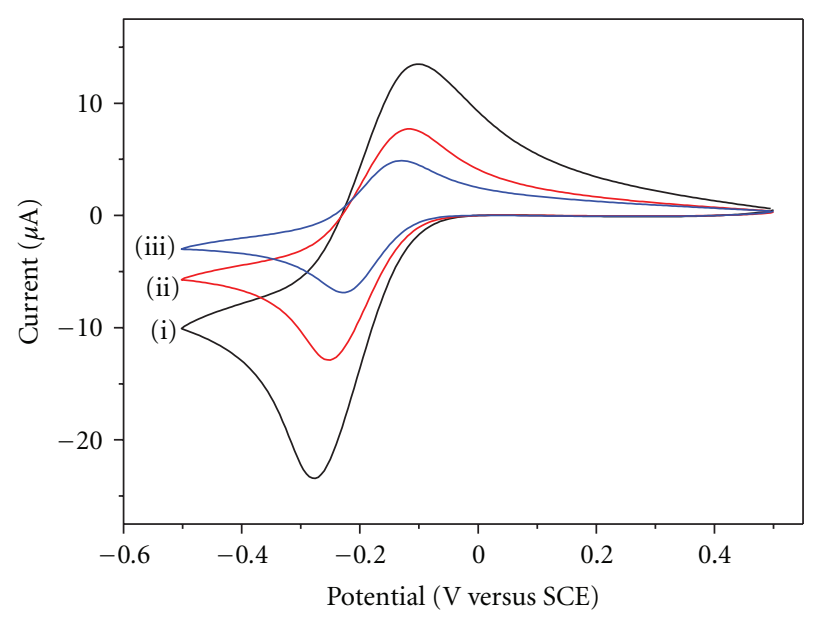

(a)

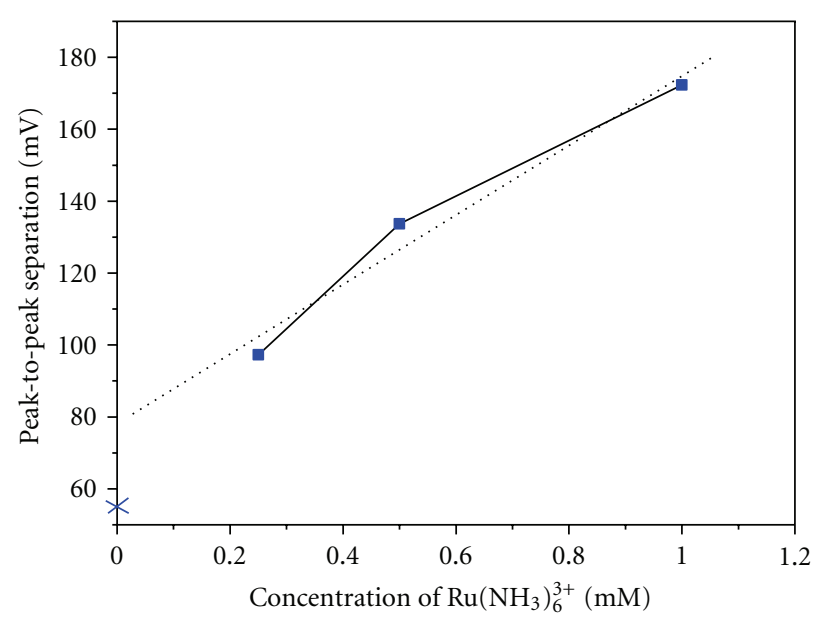

(c)

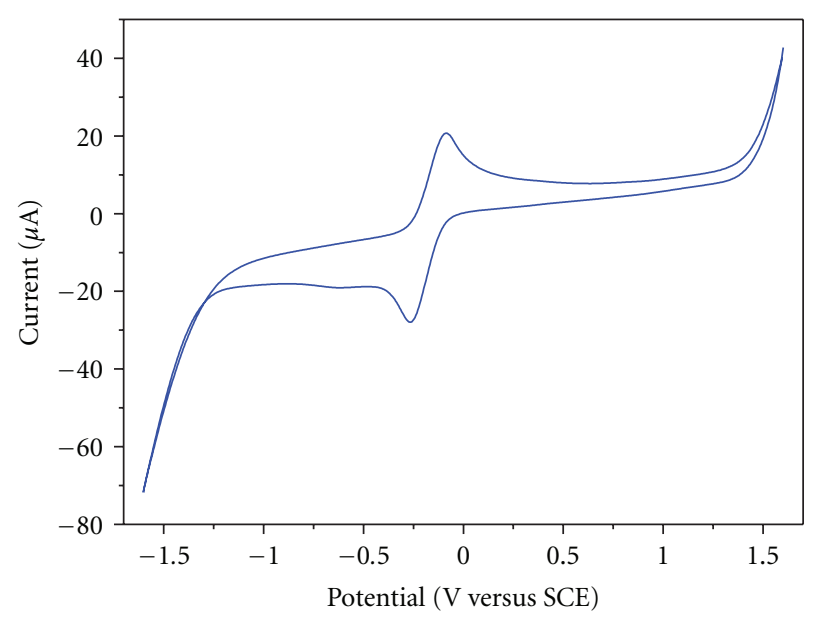

(b)

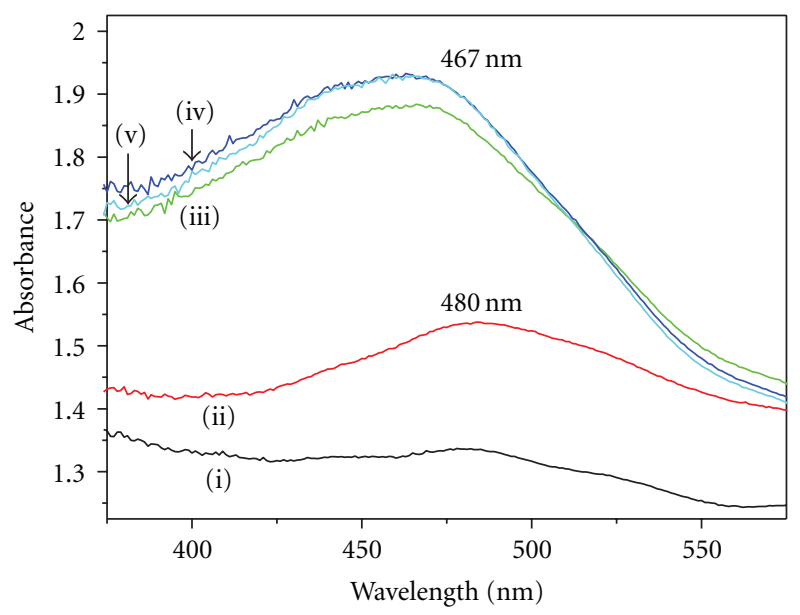

(d)

FIgURE 5: (a) Background corrected cyclic voltammograms of diamond hybrid OTE in $\mathrm{Ru}\left(\mathrm{NH}_{3}\right)_{6}^{3+}$ of different concentrations in $0.1 \mathrm{M}$ acetate buffer: (i) $1000 \mu \mathrm{M}$, (ii) $500 \mu \mathrm{M}$, and (iii) $250 \mu \mathrm{M}$, with scan rate of $50 \mathrm{mV} / \mathrm{s}$. (b) The cyclic voltammogram of diamond hybrid OTE in $1 \mathrm{mM} \mathrm{Ru}\left(\mathrm{NH}_{3}\right)_{6}^{3+}$ in $0.1 \mathrm{M}$ acetate buffer, scan rate $50 \mathrm{mV} / \mathrm{s}$, showing a wide potential window from -1.35 to $1.5 \mathrm{~V}$. (c) The peak-topeak separation in voltammograms with differing $\mathrm{Ru}\left(\mathrm{NH}_{3}\right)_{6}^{3+}$ concentrations for diamond hybrid OTE, with scan rate $50 \mathrm{mV} / \mathrm{s}$. The ideal Nernstian system exhibits a peak separation of $59.2 \mathrm{mV}$ which is marked as a crossing in the $Y$-axis. (d) UV-Vis spectroscopy of $1 \mathrm{mM} 4$ aminophenol in $0.1 \mathrm{M}$ acetate buffer of $\mathrm{pH} 4.6$ with a BDD OTE at differing applied potentials: (i) $0.1 \mathrm{~V}$, (ii) $0.12 \mathrm{~V}$, (iii) $0.14 \mathrm{~V}$, (iv) $0.16 \mathrm{~V}$, and (v) $0.18 \mathrm{~V}$.

electrical contact between diamond membrane, and ITO film is probably the major source of uncompensated resistance which could be improved further by more careful assembly.

The oxidisation of 4-Aminophenol to semiquinonimine was measured between $374 \mathrm{~nm}$ and $579 \mathrm{~nm}$ to demonstrate the spectroelectrochemical application of this diamond hybrid OTE, as depicted in Figure 5(d). The potential was stepped from 0.10 to $0.18 \mathrm{~V}$ in increments of $0.02 \mathrm{~V}$, and the spectrum was collected after equilibrium for $60 \mathrm{~s}$ at each potential. As the potential stepped positive, the peak at around 460 to $480 \mathrm{~nm}$, a characteristic peak of semiquinonimine [25], increases sharply until reaching saturation at $0.16 \mathrm{~V}$. The spectra suggest a quantitative conversion of 4-Aminophenol at this potential, in good agreement with the potential reported in the literature $[26,27]$.

\section{Conclusions}

A novel approach to fabricate diamond OTE is explored by combing the outstanding electrochemical properties of diamond and the electrical conductivity and optical transparency of metal oxide films. Conductive diamond was deposited directly on ITO and AZO film. XPS spectra after diamond growth reveal a reduction of the oxides to elemental metals by the hydrogen plasma and a fast evaporation of the metals due to elevated temperature during diamond growth. Although AZO film is reported to have the highest resistance to thermal decomposition and hydrogen plasma, it still cannot survive in the harsh conditions of diamond growth.

An alternative approach was explored to circumvent the degradation of metal oxide by preparing free-standing 
diamond membrane from diamond film on silicon wafer followed by selective chemical etching of silicon substrate, and integrating the membrane with an ITO substrate. AFM measurement shows a smooth surface with small diamond grain of 50 to $100 \mathrm{~nm}$, and Raman spectroscopy confirms the diamond phase purity and the presence of nanodiamond. The resulting OTE device exhibits competitive optical transparency and electrochemical properties compared with the value reported previously. The diamond membrane-ITO device shows well-defined redox peaks with a narrow peak separation in cyclic voltammograms, suggesting a quasireversible electrochemical kinetics. The spectroelectrochemical application of this hybrid device in the oxidation of 4Aminophenol was demonstrated, and the results agree with previous studies.

\section{Acknowledgments}

The authors would like to acknowledge the financial support from EPSRC with Grant no. EP/D504813/1. J. Hu acknowledges the financial support of Newton Abraham studentship.

\section{References}

[1] W. Kaim and A. Klein, Spectroelectrochemistry, RSC Publishing, Cambridge, UK, 2008.

[2] B. S. Pons, J. S. Mattson, L. O. Winstrom, and H. B. Mark, "Application of deposited thin metal films as optically transparent electrodes for internal reflection spectrometric observation of electrode solution interfaces," Analytical Chemistry, vol. 39, no. 6, pp. 685-688, 1967.

[3] A. Yildiz, P. T. Kissinger, and C. N. Reilley, "Evaluation of an improved thin-layer electrode," Analytical Chemistry, vol. 40, no. 7, pp. 1018-1028, 1968.

[4] H. B. Mark and B. S. Pons, "An in situ spectrophotometric method for observing the infrared spectra of species at the electrode surface during electrolysis," Analytical Chemistry, vol. 38, no. 1, pp. 119-121, 1966.

[5] R. W. Murray, W. R. Heineman, and G. W. O’Dom, "An optically transparent thin layer electrochemical cell," Analytical Chemistry, vol. 39, no. 13, pp. 1666-1668, 1967.

[6] R. G. Compton, J. S. Foord, and F. Marken, "Electroanalysis at diamond-like and doped-diamond electrodes," Electroanalysis, vol. 15, no. 17, pp. 1349-1363, 2003.

[7] J. K. Zak, J. E. Butler, and G. M. Swain, "Diamond optically transparent electrodes: demonstration of concept with ferri/ferrocyanide and methyl viologen," Analytical Chemistry, vol. 73, no. 5, pp. 908-914, 2001.

[8] S. Haymond, J. K. Zak, Y. Show, J. E. Butler, G. T. Babcock, and G. M. Swain, "Spectroelectrochemical responsiveness of a freestanding, boron-doped diamond, optically transparent electrode toward ferrocene," Analytica Chimica Acta, vol. 500, no. 1-2, pp. 137-144, 2003.

[9] J. Stotter, J. Zak, Z. Behler, Y. Show, and G. M. Swain, "Optical and electrochemical properties of optically transparent, boron-doped diamond thin films deposited on quartz," Analytical Chemistry, vol. 74, no. 23, pp. 5924-5930, 2002.

[10] Y. R. Zhang, Y. Kato, S. Yoshihara, and T. Watanabe, "A novel boron-doped diamond (BDD)-coated platinum mesh electrode for spectroelectrochemistry," Journal of Electroanalytical Chemistry, vol. 603, no. 1, pp. 135-141, 2007.

[11] G. Haacke, "New figure of merit for transparent conductors," Journal of Applied Physics, vol. 47, no. 9, pp. 4086-4089, 1976.
[12] F. Zhu, C. H. A. Huan, K. Zhang, and A. T. S. Wee, "Investigation of annealing effects on indium tin oxide thin films by electron energy loss spectroscopy," Thin Solid Films, vol. 359, no. 2, pp. 244-250, 2000.

[13] M. J. Alam and D. C. Cameron, "Preparation and properties of transparent conductive aluminum-doped zinc oxide thin films by sol-gel process," Journal of Vacuum Science and Technology a-Vacuum Surfaces and Films, vol. 19, no. 4, pp. 1642-1646, 2001.

[14] G. K. Paul and S. K. Sen, "Sol-gel preparation, characterization and studies on electrical and thermoelectrical properties of gallium doped zinc oxide films," Materials Letters, vol. 57, no. 3, pp. 742-746, 2002.

[15] Y. Avigal and A. Hoffman, "A new method for nucleation enhancement of diamond," Diamond and Related Materials, vol. 8, no. 2-5, pp. 127-131, 1999.

[16] L. Raniero, A. Gonçalves, A. Pimentel et al., "Influence of hydrogen plasma on electrical and optical properties of transparent conductive oxides," in Proceedings of the Materials Research Society Symposium, vol. 862, p. A21.10, April 2005.

[17] S. Major, S. Kumar, M. Bhatnagar, and K. L. Chopra, "Effect of hydrogen plasma treatment on transparent conducting oxides," Applied Physics Letters, vol. 49, no. 7, pp. 394-396, 1986.

[18] B. M. Ataev, A. M. Bagamadova, V. V. Mamedov, and A. K. Omaev, "Thermally stable, highly conductive, and transparent $\mathrm{ZnO}$ layers prepared in situ by chemical vapor deposition," Materials Science and Engineering B, vol. 65, no. 3, pp. 159163, 1999.

[19] R. G. Gordon, "Criteria for choosing transparent conductors," MRS Bulletin, vol. 25, no. 8, pp. 52-57, 2000.

[20] J. Cho, KI. H. Yoon, M. S. Oh, and W. K. Choi, "Effects of $\mathrm{H}$ annealing treatment on photoluminescence and structure of $\mathrm{ZnO}: \mathrm{Al} / \mathrm{AlO}$ grown by radio-frequency magnetron sputtering," Journal of the Electrochemical Society, vol. 150, no. 10, pp. H225-H228, 2003.

[21] J. Mass, P. Bhattacharya, and R. S. Katiyar, "Effect of high substrate temperature on $\mathrm{Al}$-doped $\mathrm{ZnO}$ thin films grown by pulsed laser deposition," Materials Science and Engineering B, vol. 103, no. 1, pp. 9-15, 2003.

[22] R. P. Eischens, W. A. Pliskin, and M. J. D. Low, "The infrared spectrum of hydrogen chemisorbed on zinc oxide," Journal of Catalysis, vol. 1, no. 2, pp. 180-191, 1962.

[23] N. Wada, P. J. Gaczi, and S. A. Solin, "'Diamond-like" 3-fold coordinated amorphous carbon,” Journal of Non-Crystalline Solids, vol. 35-36, no. 1, pp. 543-548, 1980.

[24] S. Michaelson, R. Akhvlediani, and A. Hoffman, "Preparation and properties of sub-micron thick and free-standing diamond membranes," Diamond and Related Materials, vol. 11, no. 3-6, pp. 721-725, 2002.

[25] J. Schwarz, W. Oelßner, H. Kaden, F. Schumer, and H. Hennig, "Voltammetric and spectroelectrochemical studies on 4-aminophenol at gold electrodes in aqueous and organic media," Electrochimica Acta, vol. 48, no. 17, pp. 2479-2486, 2003.

[26] M. Goto, M. Kato, and D. Ishii, "Semidifferential electroanalysis with a solid working electrode," Analytica Chimica Acta, vol. 126, no. C, pp. 95-104, 1981.

[27] M. Jamal, A. S. Sarac, and E. Magner, "Conductive copolymermodified carbon fibre microelectrodes: electrode characterisation and electrochemical detection of p-aminophenol," Sensors and Actuators B, vol. 97, no. 1, pp. 59-66, 2004. 


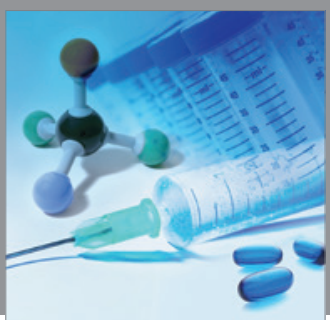

International Journal of

Medicinal Chemistry

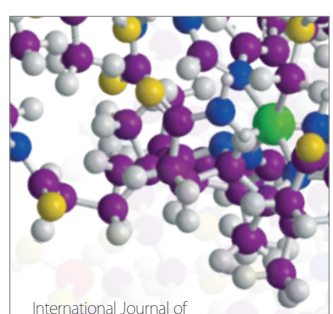

Carbohydrate Chemistry

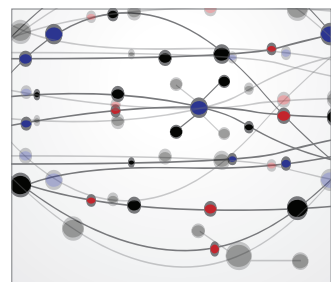

The Scientific World Journal
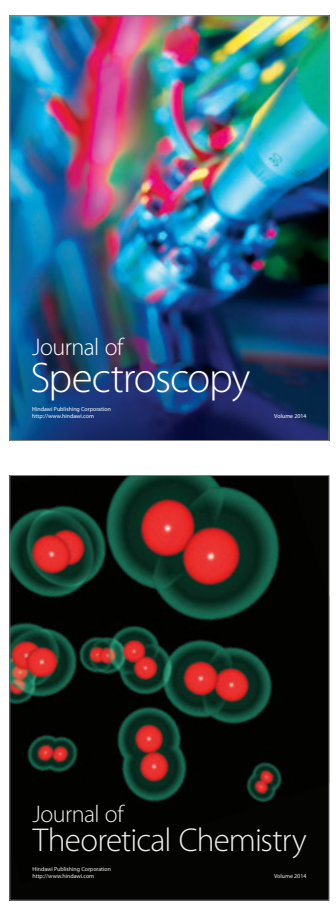
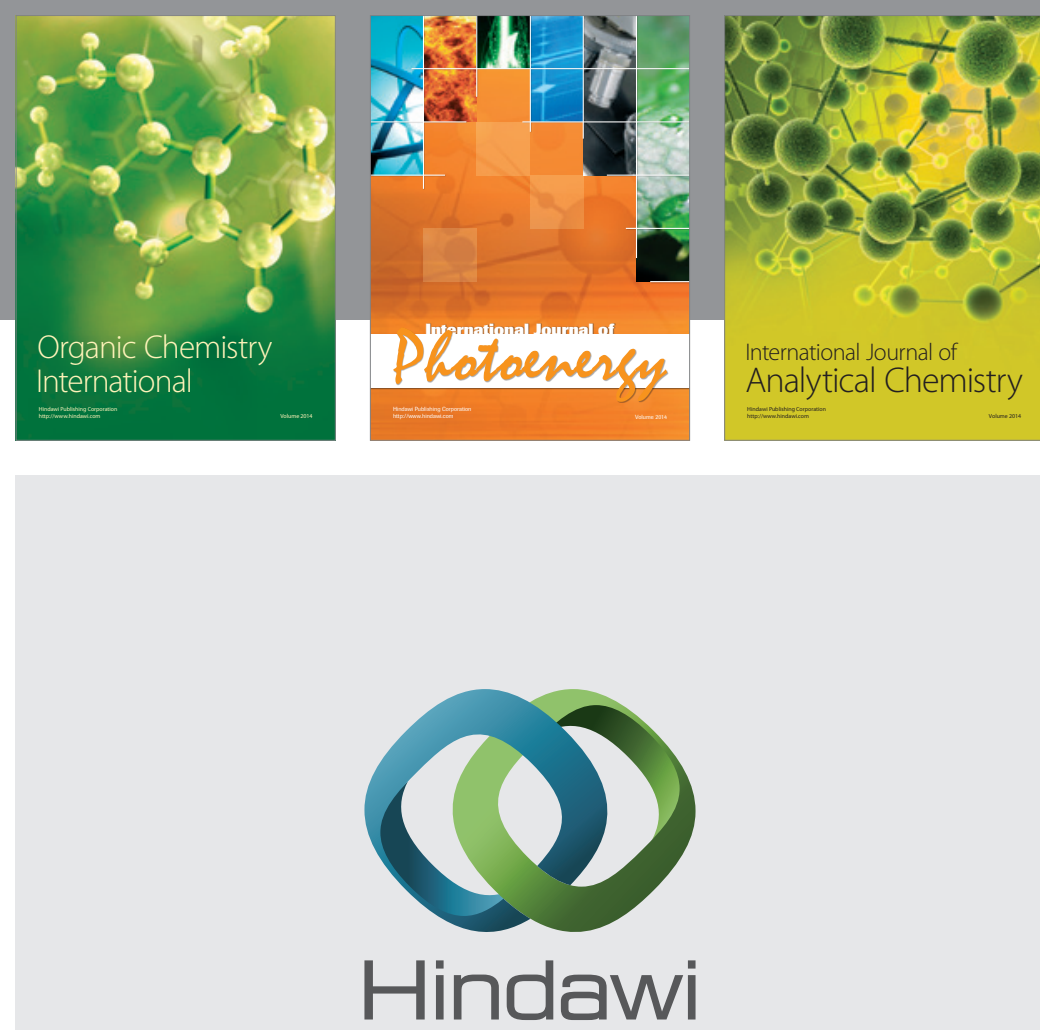

Submit your manuscripts at

http://www.hindawi.com
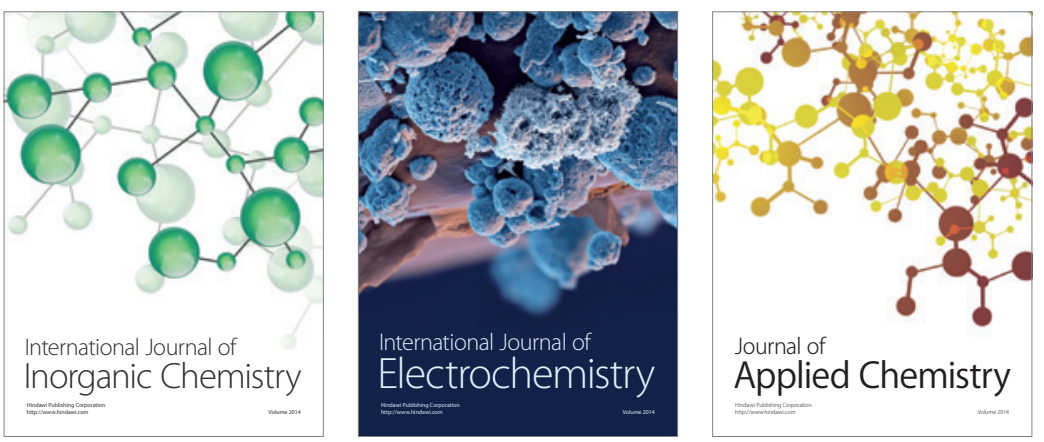

Journal of

Applied Chemistry
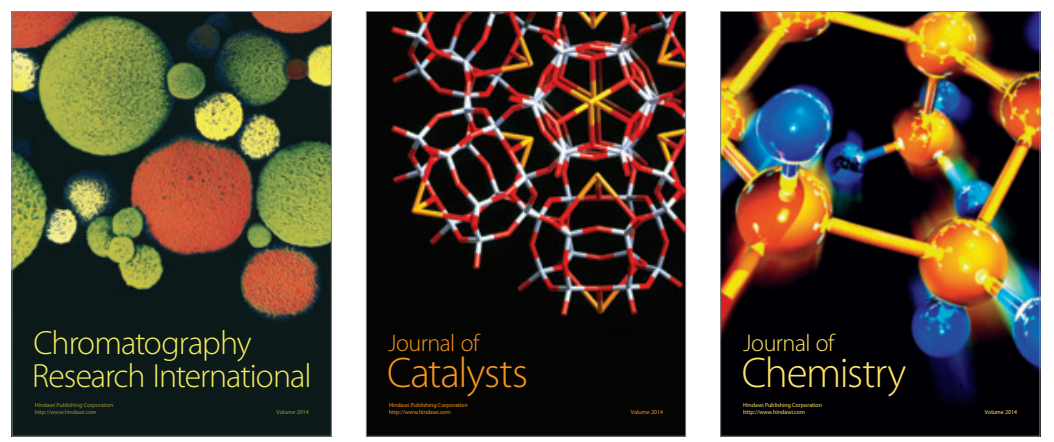
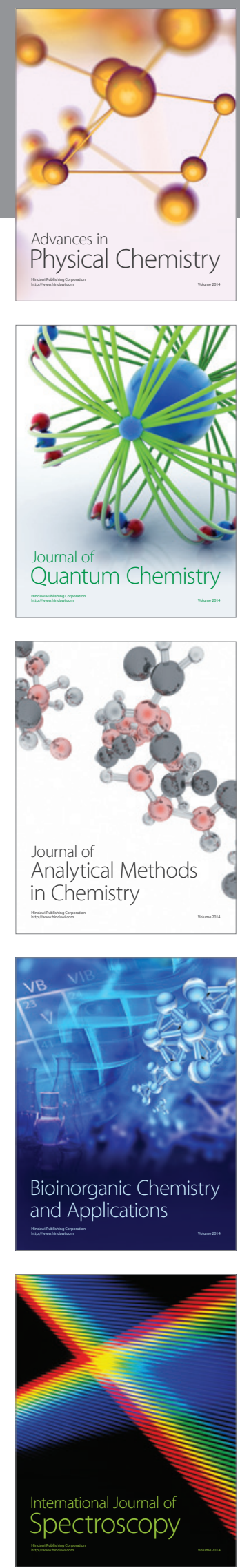